\title{
Video-assisted-thoracoscopic surgery in left-to-right Nuss procedure for pectus excavatum for prevention of serious complications - technical aspects based on 1006 patients
}

\author{
Krystian Pawlak, Łukasz Gąsiorowski, Piotr Gabryel, Wojciech Dyszkiewicz \\ Department of Toracosurgery, Poznan University of Medical Sciences, Poznan, Poland
}

Videosurgery Miniinv 2018; 13 (1): 95-101

DOI: https://doi.org/10.5114/wiitm.2018.72683

\begin{abstract}
Abstarct
Introduction: Additional use of the video-assisted thoracoscopic surgery (VATS) technique in the Nuss procedure has been globally accepted for the improvement of safety of surgical treatment as well as for decreased frequency of serious intraoperative and postoperative complications.

Aim: To evaluate VATS in surgical treatment of patients with pectus excavatum by the left-to-right Nuss procedure for prevention of serious intra- and postoperative complications.

Material and methods: From 2002 to 2016, 1006 patients with pectus excavatum aged 7 to 62 years (mean: 18.6) underwent the Nuss procedure. There were 796 males and 210 females. The clinical records of all patients were analyzed retrospectively. The follow-up varied from 1 to 172 months (mean: $80.7 \pm 43$ ).

Results: The early 30-day postoperative mortality was zero. Early thoracoscopy-dependent postoperative complications, the majority transient and non-life-threatening, occurred in $35.6 \%$ of patients. The most frequent complication was pneumothorax, diagnosed in $24.5 \%$ of patients. Two patients required repeat surgery. One patient required VATS pleurectomy due to persistent postoperative air leakage. In another patient left thoracotomy following bleeding from the pleural cavity was performed.

Conclusions: The use of VATS in the left-to-right Nuss procedure for pectus excavatum ensures the safety of surgical treatment and minimizes the occurrence of serious intra- and postoperative complications concerning injury of the mediastinum, lung, diaphragm or abdominal cavity.
\end{abstract}

Key words: Nuss procedure, postoperative complications, pectus excavatum, thoracoscopic surgery.

\section{Introduction}

Surgical treatment using the Nuss procedure has become the treatment of choice for patients with pectus excavatum. Additional use of the video-assisted-thoracoscopic surgery (VATS) technique in this procedure has been globally accepted for the improvement of safety of surgical treatment as well as for decreased frequency of serious intraoperative and postoperative complications. However, thoraco- scopy was not described in the primary Donald Nuss report in 1998 [1]. Bufo et al. were the first to report on the use of VATS for prevention of life-threatening intraoperative lesions of the mediastinum [2]. Currently, the discussion is focused on superiority of left-, right or bilateral thoracoscopic access, necessity of $\mathrm{CO}_{2}$ insufflation or sternal elevation to improve the safety during a surgical procedure [36]. Although serious life-threatening complications after the Nuss procedure are rare [7-9], VATS used

\section{Address for correspondence}

Krystian Pawlak MD, Department of Toracosurgery, Poznan University of Medical Sciences, 62 Szamarzewskiego St, $60-569$ Poznan,

Poland, phone: +48 509835 550, e-mail: krystianp@hotmail.com 
during the operation may further reduce the perioperative risk.

\section{Aim}

The aim of this study was to evaluate VATS in surgical treatment of patients with pectus excavatum by the Nuss procedure for prevention of serious intra- and postoperative complications.

\section{Material and methods}

From July 2002 to September 2016 at our Department of Thoracic Surgery, 1006 patients with pectus excavatum aged 7 to 62 years (mean: $18.6 \pm 5.7$ ) underwent the Nuss procedure. The patients included 796 (79.1\%) males and 210 (20.9\%) females. The clinical records of all patients were analyzed retrospectively. Apart from the psychological aspects of the disease, subjective symptoms were presented by 437 (43.4\%) patients and were as follows: low exercise tolerance $(n=378)$, chest pain $(n=106)$ and arrhythmias $(n=8)$. Comorbidities were observed in $101(10.0 \%)$ patients. The most frequent coexisting diseases were bronchial asthma $(n=32)$, arterial hypertension ( $n=9$ ), epilepsy $(n=8)$, mitral valve incompetence $(n=7)$ and previously operated scoliosis $(n=6)$. In 1005 (99.9\%) patients, a congenital deformity of the chest was diagnosed. In 44 (4.4\%) of those patients, a recurrent pectus excavatum after previously performed ineffective correction, usually by the Ravitch method, was diagnosed.

Prior to the surgery, routine blood tests, electrocardiography, chest X-ray and spirometry were performed in all studied patients. Among spirometry parameters, the following were evaluated in all patients: forced expiratory volume in $1 \mathrm{~s}\left(\mathrm{FEV}_{1}\right)$ ranged from $52.3 \%$ to $122.9 \%$ of the expected value (mean: $93.4 \pm 13.8 \%$ ); forced vital capacity (FVC) ranged from

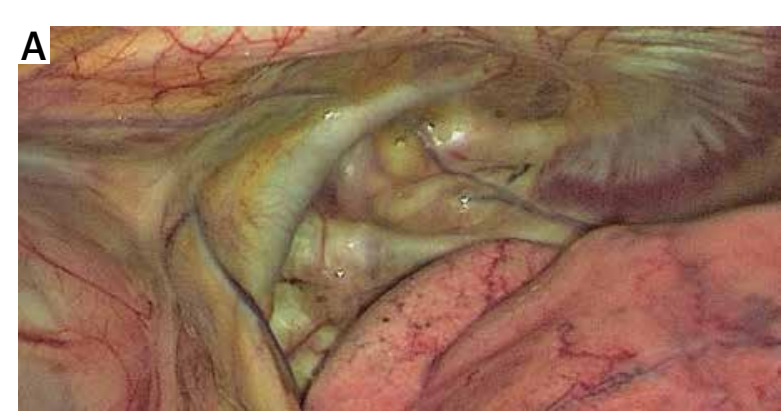

$52.4 \%$ to $124.8 \%$ of the expected value (mean: 88.7 $\pm 13.8 \%$ ); and the Tiffeneau index ranged from $71.9 \%$ to $120.4 \%$ (mean: $101.4 \pm 10.5 \%$ ). Symmetrical deformity was diagnosed in 665 (66.1\%) patients, whilst asymmetrical deformity occurred in 341 (33.9\%) cases. Asymmetrical deformity was defined when the lowest point of sternal depression was located outside of the midline of the chest. The size of the pectus excavatum was evaluated according to the Haller index based on two projection chest X-rays postero-anterior and right lateral. In our study, the Haller index ranged from 2.6 to 17.3 with a mean of $3.7 \pm 1.4$. Computed tomography (CT) scan and echocardiography were not routinely performed in our patients and indications for these examinations were limited to advanced symmetrical or significantly asymmetrical deformities. Prior to the surgery, all patients were informed about the surgical procedure and signed a consent form. Because of the retrospective nature of the study, the institutional review board permission of a local university was not required.

\section{Operative technique}

The operative technique of the Nuss procedure was mainly described in our previous report [10]. $\mathrm{CO}_{2}$ insufflation was used in none of our patients during the Nuss procedure. Instead of this hypoventilation of the lungs achieved by decreased volume of lung ventilation and increased frequency of breathing was performed to create the pneumothorax (Photos $1 \mathrm{~A}, \mathrm{~B})$. A $10 \mathrm{~mm}$, 0-degree thoracoscope (HOPKINS - Karl Storz GmbH \& Co. KG, Germany) was introduced to the left pleural cavity through the left operative wound in the anterior axillary line (Photo 2). In 13 (1.3\%) patients with advanced or significantly asymmetrical deformities, the traction of the left seventh rib cartilage using a hook device inserted at the level of the xyphoid was performed to

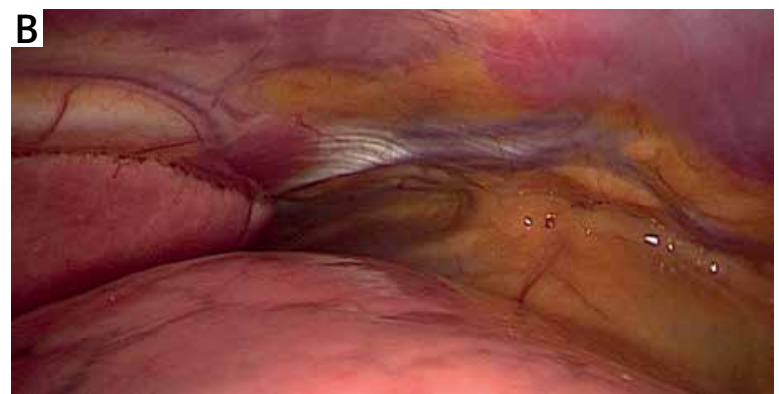

Photo 1 A, B. Collapsed lung achieved by hypoinflation of both lungs allows creation of enough free space to control retrosternal introduction of the introducer 
elevate the sternum. The steel introducer (Micromed BHH Ltd., Poland) was carried under the control of bilateral thoracoscopy through the left operative wound from the left to the right pleural cavity by blunt dissection of the retrosternal space (Photos 3 A-D). The metal corrective bar (Micromed BHH Ltd., Poland) was selected considering the size of the chest. One bar was implanted in 452 (44.9\%) patients, two bars were inserted into 545 (54.2\%) patients, whilst three bars were inserted into $9(0.9 \%)$ patients. After the bar implantation, bilateral thoracoscopy was performed to evaluate the proper location of implants, as well as the retrosternal space and sternum, pericardium, lung and diaphragm to rule out any potential injuries (Photo 4). In case of any bleeding perceived in the pleural cavity (only in 3 cases), immediate coagulation under the control of thoracoscopy was performed. In the last 555 (55.2\%) patients, a routine chest tube was inserted into the right pleural space at the final stage of the operation usually for the next 2 consecutive days. The length of postoperative hospitalization ranged from 3 to 20
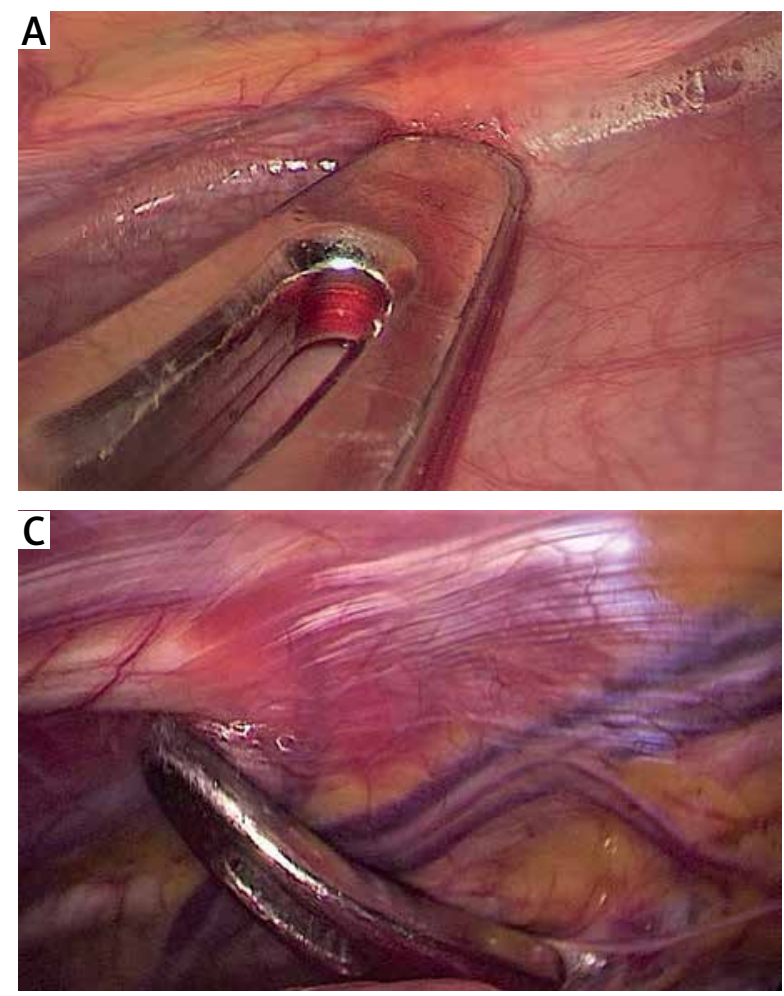

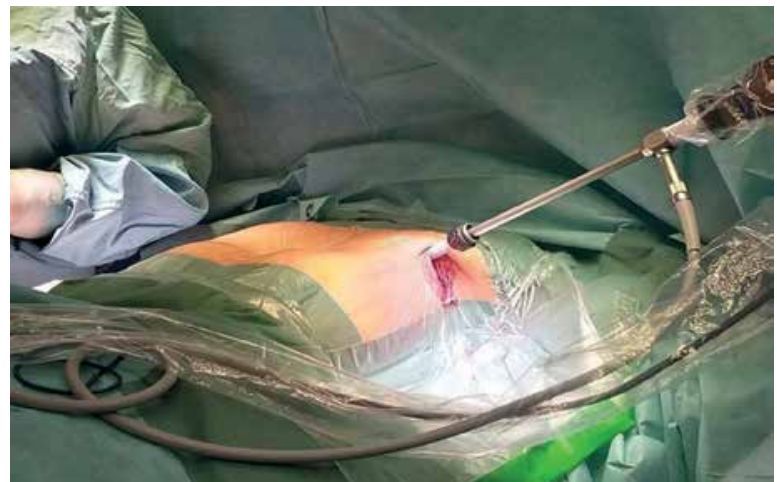

Photo 2. The thoracoscope was introduced to the left pleural cavity through the left operative wound in the anterior axillary line

days (mean: $6.3 \pm 2.0$ ). The follow-up varied from 1 to 172 months (mean: $80.7 \pm 43$ ).

\section{Results}

The length of the surgical procedure ranged from 15 to $200 \mathrm{~min}$ with a mean value of $51.5 \pm 18.6 \mathrm{~min}$.
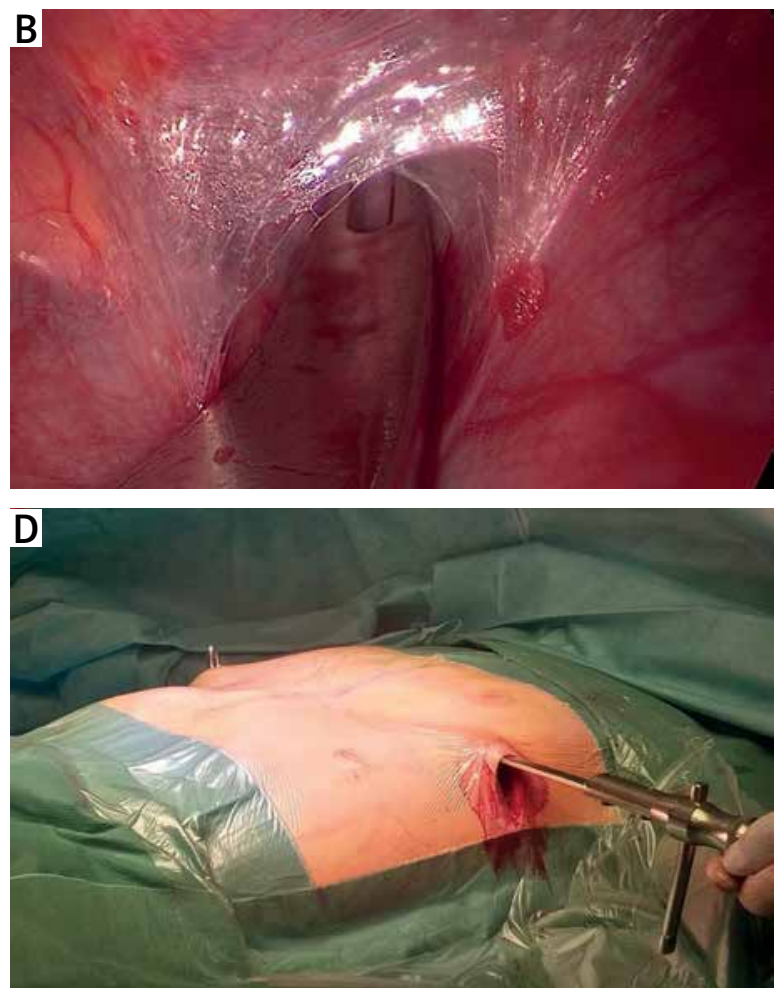

Photo 3. The introducer reaches the posterior surface of the sternum in the left pleural cavity (A) and further is moved through the mediastinal pleura in the retrosternal space (B), next goes across the right pleural space (C) and finally exits the chest through the right intercostal space and the right operative wound (D) 


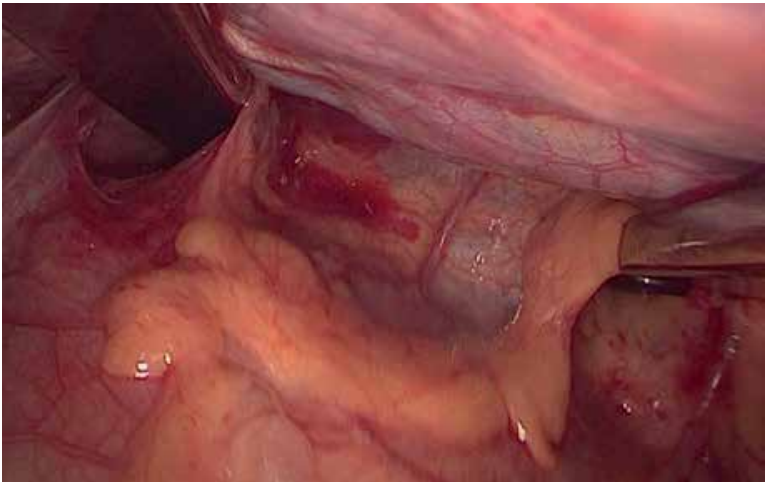

Photo 4. The position of implanted bars in the right pleural space as well as retrosternal space and mediastinum is controlled by thoracoscopy

None of the operated patients died within 30 postoperative day. The early (up to 30 days) thoracoscopy-dependent postoperative complications were in the majority of cases transient and occurred in 358 (35.6\%) patients. The most frequent complication was pneumothorax, which was diagnosed in 244 (24.5\%) patients. However, in 74 (30.3\%) of those patients, an additional pleural drainage was necessary. In the remaining patients, the pneumothorax resolved spontaneously and did not require additional surgical intervention. One patient required repeated surgery - VATS pulmonary wedge resection and pleurectomy due to persistent postoperative air leakage.

Hemothorax was observed in 13 (1.3\%) patients in the early postoperative period. Seven of those patients required repeated pleural drainage.

Among $8(0.8 \%)$ patients with anemia due to postoperative hemothorax in whom blood transfusion of more than 1 unit was needed, 1 female patient also required reoperation (left thoracotomy) following bleeding from the pleural cavity.

\section{Discussion}

Although previous reports concerning the use of thoracoscopy in the Nuss procedure indicated a significant influence of VATS on improving the safety of the surgical treatment, they were based on a relatively small number of patients [3-6]. In our study, the clinical records of 1006 patients with pectus excavatum operated on by the Nuss procedure in the last 14 years were analyzed. Currently, the discussion on the use of VATS during the Nuss procedure is focused on the most appropriate surgical access (left-, right- or bilateral thoracoscopy), the necessity of $\mathrm{CO}_{2}$ insufflation or the need of sternal elevation. The advocates of right thoracoscopy, which was also recommended in the second report of Donald Nuss in 2002, reported on safe preparation of the mediastinum in right-to-left bar insertion and appropriate evaluation of the retrosternal space, which is not limited by the silhouette of the heart. Moreover, the anteriorly bent end of the steel introducer does not expose the heart to injury $[4,6,11]$. On the other hand, in the opinion of the other authors who used left thoracoscopy with left-to-right shunt, the heart and diaphragm do not disturb the proper assessment of the route of the introducer $[3,5]$. In that approach, the sternal elevation recommended by Shaw et al. is not necessary as a routine management [3, $5,12]$. We used VATS with left-to-right shunt in all our patients, and in the majority of them we had no difficulties with evaluation of the retrosternal space exposure as well as safe transfer of the introducer. Only in 13 of our patients with advanced or significantly asymmetrical, particularly one-sided deformities, was the traction of the anterior chest by the seventh left rib cartilage in a way similar to that described by Rygl et al. necessary [13]. All of our patients since the beginning of our practice in 2002 have been operated on with the left-to-right Nuss procedure. We have not modified the operative technique apart from routine pleural drainage which has been used during the Nuss procedure in the recent half of our patients to reduce the frequency of postoperative pneumothorax as well as to control possible postoperative bleeding. Bilateral thoracoscopy has been introduced to assess the proper location of the metal bars and to look for possible bleeding in retrosternal and pleural spaces.

In our opinion, hypoinflation of the lungs minimal enough to maintain normal ventilation parameters allows sufficient sparing of retrosternal space for appropriate evaluation of the pleural cavity and the mediastinum. The hypoinflated lung collapses enough for a safe and comfortable operation. We agree with Hendrickson et al. and Palmer et al. that left thoracoscopy has the advantage over right thoracoscopy and does not increase the risk of heart injury $[3,5]$. Most of the heart mass is located in the left pleural cavity and its proper intraoperative evaluation is crucial for suitable insertion of the introducer and for avoidance of mediastinal injury. In our opinion, the introducer should be inserted 
through the left intercostal space under constant thoracoscopic visualization to avoid pericardial tear or cardiac perforation. When the introducer is inserted through the right pleural cavity, we do not have proper anticipation of the pericardium and diaphragm in the left pleural space, which may be obscured by a depressed sternum.

Some authors use insufflation of $\mathrm{CO}_{2}$ during the Nuss procedure to create an artificial pneumothorax and thereby increase the empty pleural space. They regard this method as necessary in case of the lack of selective lung ventilation [1, 3, 6, 9, 14]. Although all our patients were intubated by a standard single endotracheal tube, we did not come across any expanded lung or elevated diaphragm that would cover the operative space and complicate the surgery. This was achieved by us mainly due to hypoinflation, which causes the lungs to collapse. A similar opinion was presented by Cheng et al. [4]. Moreover, the use of $\mathrm{CO}_{2}$ insufflation can be one of the possible ways to increase the pleural space; on the other hand, it can also lead to circulatory disturbances (decrease of venous inflow or blood pressure, decreased left ventricular output or carbon dioxide embolism), which could increase the potential perioperative risk [6]. Although $\mathrm{CO}_{2}$ insufflation is used by some pediatric surgeons as well as thoracic surgeons in pectus corrective surgery, we could not find any randomized data comparing those two refinements of the Nuss procedure. In our opinion, maintaining low tidal volumes intraoperatively and thus facilitating the total re-expansion of the lungs on PEEP in the final stage of surgery is sufficient for both safe visualization and complete removal of the air from the pleural cavity. Whether to use the insufflation to improve insight visualization depends on personal experience rather than objective study.

The appropriate insertion of a thoracoscopic camera can be an important factor for the safety of the surgical procedure. Saxena et al. and Nuss et al. advocate the location of the trocar in the mid-axillary line to be behind the standard VATS approach $[6,15]$; however, in our opinion, this is insufficient because the view is obstructed by the heart, lung or diaphragm, particularly in advanced asymmetrical deformities. We inserted the trocar into the pleural space at the anterior axillary line in front of the heart and collapsed lung. This allows us to avoid the anatomical mediastinal structures and achieve enough free operative space. A slow preparation of the me- diastinum closest to the posterior sternal surface is sufficient for the safety of the procedure. Nuss et al. recommended the insertion of the trocar through the intercostal space beneath the wound [15]. Although, in our study, the trocar and introducer were inserted only through the operative wound, we did not encounter any camera-introducer conflict during the surgical procedure.

The 0-degree thoracoscope was used in all our procedures. However, although many reports indicate the need to use 30- or 45-degree thoracoscopic visualization, we believe that a direct view is enough for a proper evaluation of the small operative space and for correct positioning of the bars in Nuss procedures $[3,6,11]$. The need for direct visualization during Nuss procedures is the most frequent situation, so in our opinion, the 0-degree scope is sufficient for proper visualization of the operative field. The indirect view of the 30- or 45-degree scope may be used for more sophisticated VATS procedures of other therapeutic indications.

Although most authors emphasize that the Nuss procedure is safe and there is only a small percentage of early postoperative complications, some serious, life-threatening, even lethal complications after this minimally invasive corrective surgery have been reported. Despite the routine use of thoracoscopy, cardiac perforation [7-9, 16, 17], pericardial tear [14], pericardial tamponade $[4,18,19]$, lung injury [20], diaphragmatic liver piercing injury [6, 15, 21], vena cava inferior occlusion $[22,23]$ and internal thoracic artery disruption have been observed [24]. Castellani et al. and Erşen et al. successfully repaired intraoperative heart injury by urgent thoracotomy $[16,17]$, On the other hand, 3 cases of early postoperative death due to cardiac perforation during the Nuss procedure have been reported so far [7-9]. Although most of the life-threatening complications are diagnosed intraoperatively or in the early postoperative period; some of them may also develop later, even many weeks or months after surgery [4, $18,22]$.

It is also widely reported that the perioperative risk during Nuss procedures may depend on patients' age. The risk of postoperative complications is higher in older patients over 18 year old. In our study these patients constituted $41 \%$ of the study population, but in the majority of our patients more than one corrective bar was inserted independently of the age of the patients. Therefore, in our practice 
the procedure has not been modified depending on the age of the patient. The analysis of postoperative complications during the Nuss procedure in different age groups of patients was performed in our previous paper [10]. The difference between study groups was not statistically significant.

In our study, early, in a majority transient and non-life threatening, postoperative complications were observed in $35 \%$ of patients, which was similar to other reports $[11,16]$. There was no postoperative mortality or cardiac, pericardial or diaphragmatic injuries. However, serious, life-threatening early postoperative complications concerned 2 of our patients who required repeat surgery. An air leakage due to lung injury by the thoracoscope developed in one of our patients during hospitalization and required secondary VATS wedge resection and pleurectomy. Ohno et al. described a similar case of lung injury caused by a thin $3 \mathrm{~mm}$ device; however, their patient recovered without redo surgery [20]. Our other patient required reoperation, left thoracotomy, and needed multiple blood transfusions due to bleeding from the left pleural space. Despite the performed thoracotomy, the exact source of the bleeding was not discovered. The other 7 patients with anemia due to hemothorax required more than 1 blood unit transfusion; however, they did not require reoperations. In those patients, the hemothorax developed despite careful bleeding control performed in the final stage of surgery and coagulation of visible sources of bleeding in pleural and retrosternal spaces The frequency of hemothorax was reported to be as high as 5.8\% [16]. The development of hemothorax may come from cardiac, pericardial or great vessels, lung, diaphragm, liver perforation or injuries of chest wall vessels, including intercostal and mammary arteries, or separated chest wall muscles. Proper and gentle insertion of the trocar and introducer as well as a detailed thoracoscopic evaluation of the retrosternal space, along with entry and exit points of corrective bars, may reduce the occurrence of bleeding complications.

Pneumothorax occurred in $24 \%$ of our patients and was the most frequent among the non-life-threatening early thoracoscopy-dependent complications. The frequency of this complication was reported in a wide range from $1 \%$ to $64 \%$; however, in the majority of cases, the pneumothorax spontaneously resolved and only a small percentage of patients required surgical treatment $[4,11$,
12, 16, 19]. In most of our cases, the pneumothorax was completely reabsorbed without the need for additional surgical treatment, although 74 (7.3\%) patients required additional chest drainage. In our opinion, pneumothorax may result from difficulties in intraoperative re-expansion of the previously atelectatic and hypoinflated lung due to increased pleural cavity space following corrective anterior chest wall elevation.

Although the rate of total early postoperative complications was $35.6 \%$, the majority $(68.8 \%)$ of these complications were transient pneumothorax which resolved spontaneously and did not require any additional treatment. Thus, most of our postoperative complications could be defined as a grade I according to the Clavien-Dindo classification of Surgical complications and did not have clinical significance [25].

\section{Conclusions}

The use of VATS in the left-to-right Nuss procedure for pectus excavatum ensures the safety of surgical treatment and minimizes the occurrence of serious intra- and postoperative complications concerning injury of the mediastinum, lung, diaphragm or abdominal cavity.

\section{Conflict of interest}

The authors declare no conflict of interest.

\section{References}

1. Nuss D, Kelly RE Jr, Croitoru DP, Katz ME. A 10-year review of a minimally invasive technique for the correction of pectus excavatum. J Pediatr Surg 1998; 33: 545-52.

2. Bufo AJ, Stone MM. Addition of thorascopy to Nuss pectus excavatum repair. Pediatr Endosurg Innovative Tech 2001; 5 : 159-62.

3. Hendrickson RJ, Bensard DD, Janik JS, Partrick DA. Efficacy of left thoracoscopy and blunt mediastinal dissection during the Nuss procedure for pectus excavatum. J Pediatr Surg 2005; 40: 1312-4.

4. Cheng YL, Lee SC, Huang TW, Wu CT. Efficacy and safety of modified bilateral thoracoscopy-assisted Nuss procedure in adult patients with pectus excavatum. Eur J Cardiothorac Surg 2008; 34: 1057-61.

5. Palmer B, Yedlin S, Kim S. Decreased risk of complications with bilateral thoracoscopy and left-to-right mediastinal dissection during minimally invasive repair of pectus excavatum. Eur J Pediatr Surg 2007; 17: 81-3.

6. Saxena AK, Castellani C, Höllwarth ME. Surgical aspects of thoracoscopy and efficacy of right thoracoscopy in minimally 
invasive repair of pectus excavatum. J Thorac Cardiovasc Surg 2007; 133: 1201-5.

7. Gips H1, Zaitsev K, Hiss J. Cardiac perforation by a pectus bar after surgical correction of pectus excavatum: case report and review of the literature. Pediatr Surg Int 2008; 24: 617-20.

8. Zhang DK, Tang JM, Ben XS, et al. Surgical correction of 639 pectus excavatum cases via the Nuss procedure. J Thorac Dis 2015; 7: 1595-605.

9. Schaarschmidt K, Lempe M, Schlesinger F, et al. Lessons learned from lethal cardiac injury by Nuss repair of pectus excavatum in a 16-year-old. Ann Thorac Surg 2013; 95: 1793-5.

10. Pawlak K, Gąsiorowski Ł, Gabryel P, et al. Early and late results of the Nuss procedure in surgical treatment of pectus excavatum in different age groups. Ann Thorac Surg 2016; 102: 1711-6.

11. Nuss D, Croitoru DP, Kelly RE Jr, et al. Review and discussion of the complications of minimally invasive pectus excavatum repair. Eur J Pediatr Surg 2002; 12: 230-4.

12. St Peter SD, Sharp SW, Ostlie DJ, et al. Use of a subxiphoid incision for pectus bar placement in the repair of pectus excavatum. J Pediatr Surg 2010; 45: 1361-4.

13. Rygl M, Vyhnanek M, Kucera A, et al. Technical innovation in minimally invasive repair of pectus excavatum. Pediatr Surg Int 2014; 30: 113-7.

14. Shu Q, Shi Z, Xu WZ, et al. Experience in minimally invasive Nuss operation for 406 children with pectus excavatum. World J Pediatr 2011; 7: 257-61.

15. Nuss D, Obermeyer RJ, Kelly RE. Nuss bar procedure: past, present and future. Ann Cardiothorac Surg 2016; 5: 422-33.

16. Castellani C, Schalamon J, Saxena AK, Höellwarth ME. Early complications of the Nuss procedure for pectus excavatum: a prospective study. Pediatr Surg Int 2008; 24: 659-66.

17. Erşen E, Demirkaya A, Kılıç B, et al. Minimally invasive repair of pectus excavatum (MIRPE) in adults: is it a proper choice? Videosurgery Miniinv 2016; 11: 98-104.

18. Park HJ, Lee SY, Lee CS. Complications associated with the Nuss procedure: analysis of risk factors and suggested measures for prevention of complications. J Pediatr Surg 2004; 39: 391-5.

19. Kabbaj R, Burnier M, Kohler R, et al. Minimally invasive repair of pectus excavatum using the Nuss technique in children and adolescents: indications, outcomes, and limitations. Orthop Traumatol Surg Res 2014; 100: 625-30.

20. Ohno K, Morotomi Y, Ueda M, et al. Comparison of the Nuss procedure for pectus excavatum by age and uncommon complications. Osaka City Med J 2003; 49: 71-6.

21. Marusch F, Gastinger I. Lebensbedrohliche Komplikation einer Trichterbrust-Operation nach Nuss. Zentralbl Chir 2003; 128: 981-4.

22. Nath DS, Wells WJ, Reemtsen BL. Mechanical occlusion of the inferior vena cava: an unusual complication after repair of pectus excavatum using the Nuss procedure. Ann Thorac Surg 2008; 85: 1796-8.

23. Ballouhey Q, Leobon B, Trinchero JF, et al. Mechanical occlusion of the inferior vena cava: an early complication after repair of pectus excavatum using the Nuss procedure. J Pediatr Surg 2012; 47: 1-3.

24. Vegunta RK, Pacheco PE, Wallace LJ, Pearl RH. Complications associated with the Nuss procedure: continued evolution of the learning curve. Am J Surg 2008; 195: 313-6.
25. Clavien PA, Barkun J, de Oliveira ML, et al. The Clavien-Dindo classification of surgical complications: five-year experience. Ann Surg 2009; 250: 187-96.

Received: 26.06.2017, accepted: 2.10.2017. 